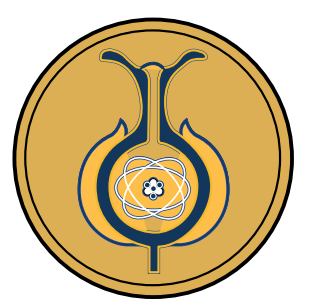

\title{
Efecto de la terapia no quirúrgica sobre parámetros clínicos periodontales en pacientes consumidores de cannabis
}

\author{
Effect of non-surgical therapy on periodontal clinical parameters in patients \\ using cannabis
}
Efeito da terapia não cirúrgica sobre parâmetros clínicos periodontais em pacientes consumidores de cannabis

\section{Natalia Gómez-Osorio ${ }^{1}$ • Paula A. González-Jiménez ${ }^{1}$ Adrián Moreno-Gutiérrez ${ }^{1}$ Danny A. Santana- Bedoya $^{1} \cdot$ Jhojan Alzate-Montoya ${ }^{1}$}

Received: Jul/14/2020 • Accepted: Aug/22/2020 • Published: Jan/31/2021

\section{Resumen}

El objetivo fue analizar los efectos de la terapia periodontal no quirúrgica sobre parámetros clínicos periodontales en pacientes consumidores de cannabis. Se realizó un estudio clínico no aleatorizado con voluntarios pertenecientes a la Universidad de Antioquia. Se revisaron 40 sujetos divididos en dos grupos: consumidores de cannabis (fumadores) y no consumidores de cannabis (control). Se registraron medidas de profundidad al sondaje (PD), nivel de inserción (CAL) y sangrado al sondaje (BOP) en el periodontograma al inicio y 3 meses después de la terapia periodontal no quirúrgica. Las diferencias entre grupos fueron analizadas con la prueba Mann Whitney. Todos los datos se analizaron en SPSS y se consideraron diferencias estadísticamente significativas cuando $p \leq 0.05$. La frecuencia del consumo de cannabis diario en pacientes fumadores evidencia que el $60 \%$ consumen 3 veces/día, el $10 \% 2$ veces/día y el $30 \% 1$ vez/día. En general, los fumadores presentaron mayor porcentaje de BOP en las dos revisiones, en comparación con el grupo de no-fumadores y esta diferencia fue estadísticamente significativa $(p<0.05)$. En profundidad al sondaje, no se observan diferencias entre los dos grupos. Los fumadores muestran un promedio de PD al inicio de 2.09 $\pm 0.73 \mathrm{~mm}$ y $2.15 \pm 0.65 \mathrm{~mm}$ a los 3 meses; el aumento fue leve entre estas. También, se observa que el nivel de inserción fue ligeramente mayor en pacientes fumadores que en no fumadores. En la población estudiada no se evidencian datos significativos asociados con enfermedad periodontal y el consumo del cannabis. La respuesta de los tejidos periodontales a la terapia no quirúrgica fue similar entre pacientes consumidores y no consumidores de cannabis.

Palabras clave: salud social; cannabis; gingivitis; profundidad al sondaje, sangrado al sondaje; nivel de inserción; terapia no quirúrgica

\footnotetext{
Natalia Gómez-Osorio, \ natalia.gomez15@udea.edu.co, (1D https://orcid.org/0000-0003-2781-7646 Paula A. González-Jiménez, $\$ paula.gonzalezj@udea.edu.co, (D) https://orcid.org/0000-0002-9967-7112 Adrián Moreno-Gutiérrez, $\square$ adrian.moreno@udea.edu.co, (D) https://orcid.org/0000-0002-2562-4560 Danny A. Santana-Bedoya, $\$ danny.santana@udea.edu.co, (1) https://orcid.org/0000-0002-2909-5045 Jhojan Alzate-Montoya, $₫$ jhojan.alzate@udea.edu.co, (D) https://orcid.org/0000-0002-0657-465X

1 Facultad de Odontología, Universidad de Antioquia, Medellín, Colombia.
} 


\section{Abstract}

The objective was to analyze the effects of non-surgical periodontal therapy on clinical parameters in patients using cannabis. A non-randomized clinical study was conducted with 40 volunteers from Universidad de Antioquia divided into 2 groups: 20 participants using cannabis (smokers) and 20 non-users (control). All clinical parameters regarding probing depth (PD), clinical attachment level (CAL), and bleeding on probing (BOP) were recorded at baseline and 3 months after non-surgical periodontal therapy. Differences between groups were assessed with the Mann Whitney test and were considered significant when $p \leq 0.05$. Data was analyzed using the SPSS statistical software. Participants using cannabis had a frequency of use of 3 times/day (60\%), 2 times/day (10\%) and 1 time/day (30\%). In general, smokers had higher BOP at both examinations as opposed to non-smokers, this difference being statistically significant $(p<0.05)$. No differences were observed for PD with a mean of $2.09 \pm 0.73 \mathrm{~mm}$ at baseline and $2.15 \pm 0.65 \mathrm{~mm}$ at 3 months, showing a small increase between the two. CAL was also slightly higher in smokers than in non-users. There was no significant evidence in the studied population relating periodontal disease with cannabis consumption. The response of periodontal tissues to non-surgical therapy was similar between cannabis users and non-users.

Keywords: Social health; cannabis; gingivitis; probing depth; bleeding on probing; clinical attachment level; non-surgical therapy

\section{Resumo}

0 objetivo foi analisar os efeitos da terapia periodontal não cirúrgica sobre parâmetros clínicos periodontais em pacientes consumidores de cannabis. Foi realizado um estudo clínico não randomizado com voluntários pertencentes à Universidade da Antioquia. Foram revisados 40 indivíduos divididos em dois grupos: consumidores de cannabis (fumantes) e não consumidores de cannabis (controle). Foram registradas medidas de profundidade à sondagem (PS), nível de inserção (NCI) e sangramento à sondagem (ISS) no periodontograma no início e 3 meses após a terapia periodontal não cirúrgica. As diferenças entre grupos foram analisadas com o teste Mann Whitney. Todos os dados foram analisados no SPSS e consideradas diferenças estatisticamente significativas quando $p \leq 0,05$. A frequência do consumo diário de cannabis em pacientes fumantes evidência que $60 \%$ consumem 3 vezes/dia, $10 \% 2$ vezes/dia e $30 \% 1$ vez/dia. Em geral, os fumantes apresentaram maior porcentagem de ISS nas duas revisões, em comparação com 0 grupo de não fumantes e esta diferença foi estatisticamente significativa $(p<0,05)$. Na profundidade à sondagem, não se observam diferenças entre os dois grupos. Os fumantes mostram uma média de PS no início de 2,09 0,73 $\mathrm{mm}$ e 2,15 0,65 $\mathrm{mm}$ aos 3 meses; 0 aumento foi leve entre estas. Observa-se, também que o nível de inserção foi ligeiramente maior em pacientes fumantes que em não fumantes. Na população estudada não se evidenciam dados significativos associados à doença periodontal e ao consumo do cannabis. A resposta dos tecidos periodontais à terapia não cirúrgica foi semelhante entre pacientes consumidores e não consumidores de cannabis.

Palavras-chave: saúde social; cannabis; gengivites; sangramento à sondagem; nível de inserção; terapia não cirúrgica 


\section{INTRODUCCIÓN}

La gingivitis es la inflamación de la encía ocasionada, principalmente, por la acumulación de placa bacteriana en la superficie dental adyacente al margen gingival. En consecuencia, la inflamación gingival se manifiesta con enrojecimiento, aumento del tamaño, cambio de la forma y sangrado al sondaje o a estímulos mecánicos como el cepillado (Espejel, Martínez, Delgado, Guzmán y Monterde, 2002). La gingivitis es la etapa precursora de la periodontitis. Esto quiere decir que, al no ser tratada o prevenida, puede avanzar a periodontitis (lesión avanzada) (Bascones y Figuero, 2005). Esta última, a diferencia de la primera, afecta directamente los tejidos de sostén periodontal y el resultado de la inflamación trae como consecuencia la pérdida de inserción del tejido conectivo, el ligamento periodontal y el hueso alveolar. Al no tratar debidamente la inflamación, esta provoca, inicialmente, bolsas periodontales y pérdida ósea. Por lo tanto, la manifestación clínica de la periodontitis se caracteriza por la formación de la bolsa periodontal adicional a la gingivitis ya establecida (Botero y Bedoya, 2010). Con el tiempo, la periodontitis puede incrementar la movilidad dental y, eventualmente, producir la pérdida dental. No obstante, el desarrollo de la enfermedad periodontal no solo depende de la placa bacteriana, también influyen otros factores importantes como el consumo de tabaco y la diabetes.

El consumo del cigarrillo ha sido ampliamente estudiado y sus efectos sobre los tejidos periodontales evaluados. Otros autores exponen que está demostrado que los pacientes fumadores presentan mayor riesgo (Odds ratio; $\mathrm{OR}>2$ ) de desarrollar periodontitis, más pérdida de inserción severa, mayor pérdida ósea y dental en una relación dependiente de la dosis y del tiempo de consumo
(Reynolds, 2014; Bouchard, Carra, Boillot, Mora y Rangé, 2017). En contraste, el análisis del consumo de cannabis y sus efectos en el periodonto no ha sido muy amplio. El THC (delta-9-tetrahidrocannabinol), con una concentración de $9.6 \%$, es el principio activo de la marihuana que, al ser inhalado, pasa al torrente sanguíneo, ejerciendo efectos en todo el cuerpo, especialmente en los sistemas cardiovascular, respiratorio e inmunológico; por lo tanto, convierte a los consumidores en personas propensas a infecciones bucales como la enfermedad periodontal (Chisini, Cademanorti y Francia, 2019; Versteeg, Slot, Van der Velden y Van der Weidjen, 2019).

La Secretaría Seccional de Salud y Protección Social de Antioquia reporta que, debido a los altos índices de consumo de marihuana en Medellín (Colombia), la cual es la tercera sustancia más consumida después del alcohol y tabaco, se hace foco de investigación dado su gran impacto en la salud de los seres humanos. Adicionalmente, se indica que el inicio del consumo ha pasado de los 16-18 años a los 12, acorde con la facilidad de acceso a tal producto (Secretaría de Salud y Protección Social, 2017). No obstante, se encuentra un vacío de información y conocimiento sobre los efectos del consumo de marihuana y su relación con enfermedad periodontal; son casi inexistentes las investigaciones en este campo, en Medellín. Por ende, el propósito de esta investigación fue analizar los efectos de la terapia periodontal no quirúrgica sobre parámetros clínicos periodontales en pacientes consumidores de cannabis.

\section{MÉTODOS}

Se realizó un estudio clínico no aleatorizado con estudiantes voluntarios pertenecientes a la Universidad de Antioquia (entre julio del 2019 y marzo del 2020). Todos los 
participantes leyeron y dieron su consentimiento de participación a través de un formato firmado y aprobado por protocolo, por el comité de ética de la Facultad de Odontología de la Universidad de Antioquia.

Muestra y selección de participantes. Se determinó una muestra no probabilística por conveniencia de 40 sujetos divididos en dos grupos: consumidores de cannabis (fumadores) y no consumidores de cannabis (control). Para la inclusión en el estudio, los participantes debían cumplir con los siguientes criterios: 18 años cumplidos o más, al menos 15 dientes en boca, intervención voluntaria y firma del consentimiento escrito informado. Adicionalmente, fueron excluidos los participantes que consumían otro tipo de sustancias psicoactivas, con menos de 15 dientes en boca, con enfermedades sistémicas no controladas (tales como diabetes, HIV, SIDA, lupus, leucemia) o mujeres en estado de embarazo. Las personas menores de 18 años también fueron excluidas de la investigación.

Los participantes consumidores de cannabis manifestaban su uso de este con fines recreativos y lo hacían por voluntad propia. En contraste, los participantes no consumidores externaron que no empleaban ningún tipo de sustancia, incluido el tabaco.

Examen clínico. El examen periodontal fue practicado por dos clínicos $(\mathrm{NG}$, PG). Para tal fin, se empleó una sonda milimetrada UNC-15, con la cual se registraron las medidas en el periodontograma correspondientes a margen gingival, profundidad al sondaje (PD), nivel de inserción periodontal (CAL) y sangrado al sondaje (BOP) excluyendo terceros molares. El examen clínico fue repetido 3 meses después de la intervención en todos los sujetos.

Intervención. Inmediatamente después del examen clínico, se realizó terapia periodontal no quirúrgica con instrumentos ultrasónicos, en todos los sujetos. La intensidad de la terapia fue dependiente de la cantidad de depósitos de placa y cálculos supragingivales y subgingivales. Finalizada la intervención, a cada paciente se le suministró un cepillo de dientes (Vitis medio, Dentaid-Colombia) y crema dental (Vitis Encías, Dentaid-Colombia), pero no se hizo ninguna modificación en la técnica de cepillado para evaluar los efectos de la terapia periodontal.

Análisis estadístico. Los datos demográficos son presentados como el promedio \pm SD y frecuencia en variables categóricas. Por su parte, los parámetros clínicos periodontales son mostrados como el promedio \pm SD (desviación estándar) o mediana IQR (rango intercuartil), dependiendo de su distribución. Para comparar los cambios de antes y después de la terapia en cada grupo, se emplearon pruebas paramétricas (matched pair $t$-test) o no paramétricas (Wilcoxon test) dependiendo de su distribución. Diferencias entre grupos fueron analizadas con la prueba Mann Whitney test. Todos los datos se analizaron en SPSS y se consideraron diferencias estadísticamente significativas cuando $\mathrm{p} \leq 0.05$.

\section{ANÁLISIS Y RESULTADOS}

En la tabla 1, se presenta la descripción demográfica de la muestra del estudio: sexo, edad, número de dientes en boca, frecuencia de consumo diario y tiempo de consumo de cannabis en años. En total, se incluyeron 40 sujetos que cumplieron con los criterios de selección, distribuidos así: 20 consumidores de cannabis (edad promedio $24.9 \pm 5$ ) y 20 no consumidores (edad promedio $23.8 \pm 2.5$ ). En los fumadores, el $90 \%$ de los pacientes atendidos fue hombres y el $10 \%$ mujeres. Por otro lado, en los no fumadores fue más alto el porcentaje de mujeres (65\%) y el $35 \%$ hombres. 
La frecuencia del consumo diario de cannabis en los pacientes fumadores evidencia que el $60 \%$ de ellos lo hacen 3 veces/día, el $10 \% 2$ veces/día y el $30 \% 1$ vez/ día. Con respecto a tiempo de consumo, se observa que la población tiene una mediana de consumo de 4.5 años [2-9.2] con un rango variable entre 2 y 9 años.

En la tabla 2, se muestra la comparación de los parámetros clínicos periodontales entre los dos grupos. En general, los sujetos fumadores presentaron mayor porcentaje de sangrado al sondaje (BOP) en las dos revisiones (T1 revisión inicial y $\mathrm{T} 2$ tres meses posterapia periodontal), en comparación con el grupo de no fumadores, y esta distinción fue estadísticamente significativa $(p<0.05)$. No hubo diferencias en el BOP entre T1 y T2, en cada grupo. En la profundidad al sondaje (PD), tampoco se observan discrepancias entre las dos agrupaciones, ya que presentan una PD similar, los fumadores muestran un promedio de PD en T1 de $2.09 \pm 0.73$ y en T2 de $2.15 \pm 0.65$; el aumento fue leve entre estas. De igual manera, no se notaron diferencias significativas en el nivel de inserción (CAL) entre los grupos, antes y después de la terapia. No obstante, se vio que el CAL fue mayor en pacientes fumadores que en no fumadores.

Tabla 1. Descripción demográfica de la muestra del estudio

\begin{tabular}{lccc}
\hline & & Fumadores & No fumadores \\
\hline Número de sujetos & & 20 & 20 \\
Edad (promedio \pm SD) & & $24.9 \pm 5$ & $23.8 \pm 2.5$ \\
Sexo & Hombres & $18(90 \%)$ & $7(35 \%)$ \\
& Mujeres & $2(10 \%)$ & $13(65 \%)$ \\
Frecuencia de consumo de cannabis & $1 /$ día & $6(30 \%)$ & NA \\
& $2 /$ día & $2(10 \%)$ & \\
Tiempo de consumo en años (mediana IQR) & $3 /$ día & $12(60 \%)$ & \\
Número de dientes en boca (promedio \pm SD) & & $4.5[2-9.2]$ & NA \\
\hline
\end{tabular}

SD: desviación estándar. NA: no aplica. IQR: rango intercuartil.

Nota: Fuente propia del estudio o la investigación.

Tabla 2. Comparación de los parámetros clínicos periodontales entre los grupos

\begin{tabular}{lccc}
\hline \multicolumn{1}{c}{ Parámetro } & Fumadores & No fumadores & P \\
\hline T1 BOP (\%) mediana [IQR] & $4.4[4.4-6.9]$ & $2.6[2.6-3.5]$ & $<0.05^{*}$ \\
T2 BOP (\%) mediana [IQR] & $5.3[4.4-6.6]$ & $2.6[1.7-2.6]$ & $<0.05^{*}$ \\
P & NS & NS & \\
T1 PD mm (promedio \pm SD) & $2.09 \pm 0.73$ & $2.04 \pm 0.57$ & NS \\
T2 PD mm (promedio \pm SD) & $2.15 \pm 0.65$ & $2.00 \pm 0.52$ & NS \\
P & NS & NS & \\
T1 CAL mm (promedio \pm SD) & $1.65 \pm 0.83$ & $1.49 \pm 0.73$ & NS \\
T2 CAL mm (promedio \pm SD) & $1.58 \pm 0.67$ & $1.44 \pm 0.69$ & NS \\
P & NS & NS & \\
\hline
\end{tabular}

IQR: rango intercuartil. SD: desviación estándar. T1: inicio. T2: 3 meses posterapia periodontal. *Mann Whitney test.

Nota: Fuente propia del estudio o la investigación. 


\section{DISCUSIÓN}

Este estudio se realizó en participantes voluntarios jóvenes mayores de 18 años; se analizan los efectos de la terapia periodontal no quirúrgica sobre parámetros clínicos periodontales en consumidores de cannabis, considerando si este era un factor de riesgo en los adultos jóvenes. Antes de exponer los hallazgos, se deben tener en cuenta algunas limitaciones que pueden explicar las diferencias con otras investigaciones, como el número de sujetos evaluados, el tiempo de reexaminación después de la terapia periodontal y el número de superficies revisadas por diente que se tuvieron en cuenta (cuatro en lugar de seis, excepto terceros molares).

Después de la revisión de la literatura, el primer trabajo en publicar la relación acerca del uso de cannabis y enfermedad periodontal fue el realizado por Thomson et al. (2008). Este estudio prospectivo fue llevado a cabo en una cohorte de sujetos nacidos entre 1972 y 1973, en el hospital Queen Mary de Nueva Zelanda y se reporta la exposición a cannabis a los 18, 21, 26 y 32 años de edad. Los participantes fueron clasificados en tres grupos: no fumadores, fumadores moderados y grandes fumadores de cannabis. Los autores reportaron una fuerte asociación entre el consumo de cannabis y periodontitis, indicando que fumar cannabis a largo plazo es perjudicial para los tejidos periodontales (Thomson et al., 2008). No obstante, los resultados deben ser considerados con cuidado durante la comparación con nuestras pesquisas, ya que se debe tener en cuenta que son poblaciones con entornos, costumbres y edades diferentes, además del tiempo entre revisiones clínicas. Otra indagación (Shariff, Ahluwalia y Papapanou, 2012), en la que participaron 980 hombres y 958 mujeres consumidores y no consumidores de cannabis, con edades entre 30 y 59 años, en los Estados Unidos, registró una asociación significativa entre el consumo de cannabis o tabaco y la afectación de los tejidos periodontales. Esto redirecciona el resultado o la comparación que se puede hacer con este estudio, ya que se tomó en cuenta también el consumo del tabaco, lo que puede inferir que los hallazgos no están claros para demostrar que el consumo de cannabis es el responsable de la pérdida de inserción en el periodonto. Por otro lado, se encuentra concordancia con lo resuelto por López y Baelum (2009), respecto a las derivaciones del actual trabajo, el cual no halló una relación significativa entre el uso de cannabis y periodontitis. Los investigadores realizaron una labor transversal en Santiago de Chile con 9163 estudiantes de edades entre los 12 y 21 años, donde se evaluó el estado periodontal de los adolescentes. Sin embargo, sugirió que el cannabis puede producir un efecto en pacientes que sean consumidores durante un mayor periodo (López y Baelum, 2009).

Al analizar los resultados logrados después de la terapia periodontal, se evidencia un aumento leve en el sangrado al sondaje en los pacientes consumidores, sin embargo, no es un determinante causal de periodontitis o pérdida de inserción en los jóvenes consumidores mayores de 18 años. Además, los estudios de referencia no consideraron el porcentaje del sangrado, por lo tanto, no es un dato comparable (Thomson et al., 2008; Shariff, Ahluwalia y Papapanou, 2012; López y Baelum, 2009).

Otro estudio sugiere que la salud periodontal en pacientes consumidores de drogas se puede ver comprometida por factores como la mala higiene, la malnutrición y el acceso limitado al cuidado dental (Kayal, Elias, Alharthi, Demyati y Mandurah, 
2014). Los resultados de esta investigación muestran un mayor sangrado al sondaje en los sujetos consumidores de cannabis, hecho que puede estar más vinculado, entonces, a la deficiente higiene bucal que al mismo consumo de cannabis.

De los trabajos revisados, teniendo en cuenta el nuestro, tres de ellos se llevaron a cabo en adultos y solo uno en adolescentes. El estudio de Shariff, Ahluwalia y Papapanou (2012) es el único que incluye pacientes con diabetes, mientras que solo el de López y Baelum (2009) ha evaluado los hábitos de higiene de los pacientes.

Aunque el ligamen entre el consumo del cannabis y la enfermedad periodontal ha arrojado diferentes resultados en los estudios, se debe continuar realizando más análisis al respecto, para así determinar una verdadera influencia o no del cannabis en los tejidos periodontales (Mederos, Francia, Chisini, Grazioli y Andrade, 2018; Serrano, Rubio, López-Pintor y Alonso, 2018). Se debe crear conciencia en el entorno de los consumidores habituales de cannabis acerca de la importancia de la salud bucal indiferente a los resultados obtenidos.

\section{CONCLUSIONES}

En la población estudiada no se evidencian datos significativos asociados con enfermedad periodontal y el consumo del cannabis. La respuesta de los tejidos periodontales a la terapia no quirúrgica fue similar entre pacientes consumidores y no consumidores de cannabis.

\section{RECONOCIMIENTOS}

Los autores agradecen a Dentaid (Colombia) por la donación de cepillos y crema de dientes para suministrar a los pacientes.

\section{DECLARACIÓN DE LA CONTRIBUCIÓN DE LOS AUTORES}

Todos los autores contribuyeron en igual proporción para la conceptualización, preparación y corrección de este artículo: $\mathrm{N}$. G. O $20 \%$, P. A. G. J. $20 \%$, A. M. G. $20 \%$, D. A. S. B. $20 \%$, J. A. M. $20 \%$.

\section{DECLARACIÓN DE DISPO- NIBILIDAD DE LOS DATOS}

Los datos que respaldan los resultados de este estudio serán puestos a disposición por el autor correspondiente [N. G. O.], previa solicitud razonable.

\section{REFERENCIAS}

Bascones, A. y Figuero, E. (2005). Las enfermedades periodontales como infecciones bacterianas. Avances en Periodoncia e Implantología Oral, 17(3), 147-156. https://doi.org/10.4321/ S1699-65852005000300004

Botero, J. E. y Bedoya, E. (2010). Determinantes del diagnóstico periodontal, Determinants of periodontal diagnosis. Revista Clínica de Periodoncia Implantología y Rehabilitación Oral, 3(2), 94-99. https://doi.org/10.1016/ S0718-5391(10)70049-5

Bouchard, P.; Carra, M. C.; Boillot, A.; Mora, F. y Rangé, H. (2017). Risk factors in periodontology: a conceptual framework. Journal of Clinical Periodontology, 44(2), 125-131. https:// doi.org/10.1111/jcpe. 12650

Chisini, L. A.; Cademartori, M. G.; Francia, A.; Mederos, M.; Grazioli, G.; Conde, M. y Correa, M. B. (2019). Is the use of Cannabis associated with periodontitis? A systematic review and meta-analysis. Journal of Periodontal Research, 54(4), 311-317. https://doi. org/10.1111/jre. 12639

Espejel, M.; Martínez, I.; Delgado, J.; Guzmán, C. y Monterde, M. (2002). Gingivitis. Revista $A D M$, 59(6), 216-219. 
Kayal, R. A.; Elias, W. Y.; Alharthi, K. J.; Demyati, A. K. y Mandurah, J. M. (2014). Illicit drug abuse affects periodontal health status. Saudi Medical Journal, 35(7), 724-728.

López, R. y Baelum, V. (2009). Cannabis use and destructive periodontal diseases among adolescents. Journal of Clinical Periodontology, 36(3), 185-189. https://doi. org/10.1111/j.1600-051X.2008.01364.X

Mederos, M.; Francia, A.; Chisini, L. A.; Grazioli, G. y Andrade, E. (2018). Influencia del consumo de cannabis en la enfermedad periodontal: una Scoping Review. Odontoestomatología, 20(31), 4-15. https://dx.doi.org/10.22592/ ode 2018 n31a2

Reynolds, M. A. (2014). Modifiable risk factors in periodontitis: at the intersection of aging and disease. Periodontology 2000, 64(1), 7-19. Doi: $10.1111 /$ prd.12047

Secretaría de Salud y Protección Social. (2017). Situación del consumo de drogas en Antioquia. Medellín.gov.co [Internet] Medellín [consultado el 9 de oct., 2017]. Recuperado de http:// studylib.es/doc/5723708/situacion-del-consumo-de-drogas-en-antioquia
Serrano, M. C.; Rubio, L.; López-Pintor, R. M. y Alonso, B. (2018). Implicaciones periodontales del abuso del cannabis. Revisión y casos clínicos. Cientifica Dental, 15(3), 173-178.

Shariff, J. A.; Ahluwalia, K. P. y Papapanou, P. N. (2012). Relationship Between Frequent Recreational Cannabis (Marijuana and Hashish) Use and Periodontitis in Adults in the United States: National Health and Nutrition Examination Survey 2011 to 2012. Journal of Periodontology, 88(3), 273-280. https://doi. org/10.1902/jop.2016.160370

Thomson, W. M.; Poulton, R.; Broadbent, J. M.; Moffitt, T. E.; Caspi, A.; Beck, J. D.; Welch, D. y Hancox, R. J. (2008). Cannabis smoking and periodontal disease among young adults. JAMA, 299(5), 525-531. https://doi. org/10.1001/jama.299.5.525

Versteeg, P. A.; Slot, D. E.; van der Velden, U. y van der Weijden, G. A. (2008). Effect of cannabis usage on the oral environment: a review. International Journal of Dental Hygiene, 6(4), 315-320. https://doi. org/10.1111/j.1601-5037.2008.00301.x

\section{(c) $(1 \otimes \Theta$}

Efecto de la terapia no quirúrgica sobre parámetros clínicos periodontales en pacientes consumidores de cannabis (Natalia Gómez-Osorio • Paula A. González-Jiménez • Adrián Moreno-Gutiérrez • Danny A. Santana-Bedoya • Jhojan Alzate-Montoya) Uniciencia is protected by Attribution-NonCommercial-NoDerivs 3.0 Unported (CC BY-NC-ND 3.0) 